\title{
Invasive Techniques in Scar Management: Fat Injections
}

F. Bassetto, C. Scarpa, and V. Vindigni

\section{Contents}

39.1 Adipose-Derived Stem Cells: Their Biological Properties - 334

39.2 The Guidelines - 334

39.3 The Procedure $-\mathbf{3 3 5}$

39.4 The Free Fat Grafting and Scars [3-9]

(• Figs. 39.5, 39.6, 39.7, and 39.8) - 336

39.4.1 The Skin Texture, Thickness, and Pliability - 339

39.4.2 The Fibrosis - 339

39.4.3 The Volume and Contour -340

39.4.4 Pain: The Analgesic Effect -340

39.4.5 The Possible Applications - 340

39.5 The Ancillary Procedures to Increase the Overall Survival of Adipose Cells - 340

39.5.1 The Plasma-Rich Platelet [10] - 340

39.5.2 The External Volume Expansion [11] - 341

39.5.3 The Future -341

39.6 Something to Discuss: The Oncological Point of View - 341

39.7 Conclusion -341

References -342 


\section{Background}

Since the end of the nineteenth century, fat grafting has become one of the most promising treatments for aesthetic and reconstructive purposes. Its very first application dates back to 1889 when Van der Mullen used fat grafting to treat a diaphragmatic hernia; some years later, in 1893, Neuber A. treated posttraumatic depressed facial scars with a small-dimension autologous fat graft taken from the forearm. Neuber, indeed, noticed that the application of small pearls of fat, compared with the bigger ones, could bring to better aesthetic results. Later, in 1910, Lexer reported the application of the autologous fat grafting for facial rejuvenation; in fact, he uses the fat as a filler for periorbital and facial wrinkles. During his experience, Lexer noted that, in order to obtain a good aesthetic results, the fat should not be damaged during the taking procedure.

In the same period, authors reported their experience with the engraftment of fat tissue, and in 1911 Brunning noticed that fat graft could be reabsorbed.

From 1912 to the Second World War, many studies have reported on the application of lipofilling for "aesthetic purpose" as treatment of the scars; but in the meantime, researchers such as Wasserman in 1926, and later Neuhof, Shapiro, and others, demonstrated that the fat grafting was a "living tissue" in which there were present preadipocytes which could later become mature adipocytes. They also noted that even if there were a degenerative process for the first months after the implantation, later there was the presence of a regenerative process with a full transformation of the preadipocytes in mature adipocytes after 5 months.

But it has been only during the end of the twentieth century that the real features and potentiality of the autologous fat graft started to become clear. In fact, it was only during that period that researchers started to demonstrate the presence of stem cells, the adipose-derived stem cells, which could survive after the transplantation and regenerate the adipose tissue. Nowadays, it is a common opinion that there are three zones that arrange the autologous fat grafting: (1) the outer one called "surviving zone," (2) the intermediate one called the "regenerating zone," and( 3 ) the inner one called the "necrotic zone."

As the name suggests, the intermediate zone or regenerating zone can replace the dead adipocytes in necrotic/center area; this process is allowed by the presence of the so-called stromal vascular fraction (SVF). The SVF indeed consists in a multiple cellular lineage composed by fibroblasts, pericytes, endothelial cells, and mesenchymal stem cells which composed the "adipose-derived stem cells" (ADSCs) and which can promote wound healing stimulating the reepithelization and neo-angiogenesis.

But how can ADSCs do this?

\subsection{Adipose-Derived Stem Cells: Their Biological Properties}

Obtained by a lipo-aspiration procedure, ADSCs can be found in large quantities, representing a fraction of $1 / 500-1 / 1500$ cells for a total of 5000 per cell each gram of fatty tissue taken, with stem potential 500 times higher than the medullary equivalent.

The adipose-derived stem cells have both a regenerative and a volumetric effect due to multiple mechanisms:

1. They produce a sort of filler effect because of the direct differentiation of the preadipocytes to mature adipocytes. This differentiation is promoted by an auto-paracrine stimulation too.

2. They have an antioxidant effect and protect against damage due to ischemia reperfusion, reactive oxygen species (ROS), and hypoxia.

3. They induce angiogenesis promoting the perfusion of the treated area, supporting existing vascular structures, and having a paracrine promotion of angiogenesis.

4. They modulate inflammation suppressing the $\mathrm{T}$ - and B-cell proliferation via NFkB-mediated mechanism; they also produce IL6 and IL8. These cytokines can recruit monocytes and macrophages in the site of injury because of their chemoattractive role.

5. They modulate the granulation tissue and other processes as fibrosis and/or reepithelization. In fact, they upregulate type I procollagen al $\mathrm{mRna}$, and they stimulate the migration of keratinocytes and fibroblasts.

6. They secrete lymphangiogenic factors.

7. They recruit systemic endogenous stem cells via a home chemokine gradient to the area of injury.

\subsection{The Guidelines}

As every procedure, the free fat grafting also has to follow some guidelines. From 2007 to 2014, the US Food and Drug Administration and the American Society of Plastic Surgeons (ASPS) and American Society for Aesthetic Plastic Surgery (ASAPS) proposed many guidelines [1, 2], but it has been only in 2016 that there have been clear guidelines. In fact, FDA defined the adipose tissue as a "structural tissue" because it is a "connective tissue that stores energy in the form of lipids, insulates the body, and provides cushioning and support for subcutaneous tissues and internal organs"; but this definition restricted its use. Nowadays, the guidelines can be summarized as follows:

1. The graft should be taken and used in the same patient at the same session. Delayed grafting is not allowed.

2. The graft should be minimally manipulated. Based on the definition given by FDA, the minimal manipulation is "a process that doesn't alter the original features of the tissue, which maintains its utilities." The graft shouldn't be sizing. Even if centrifugation is consid- 

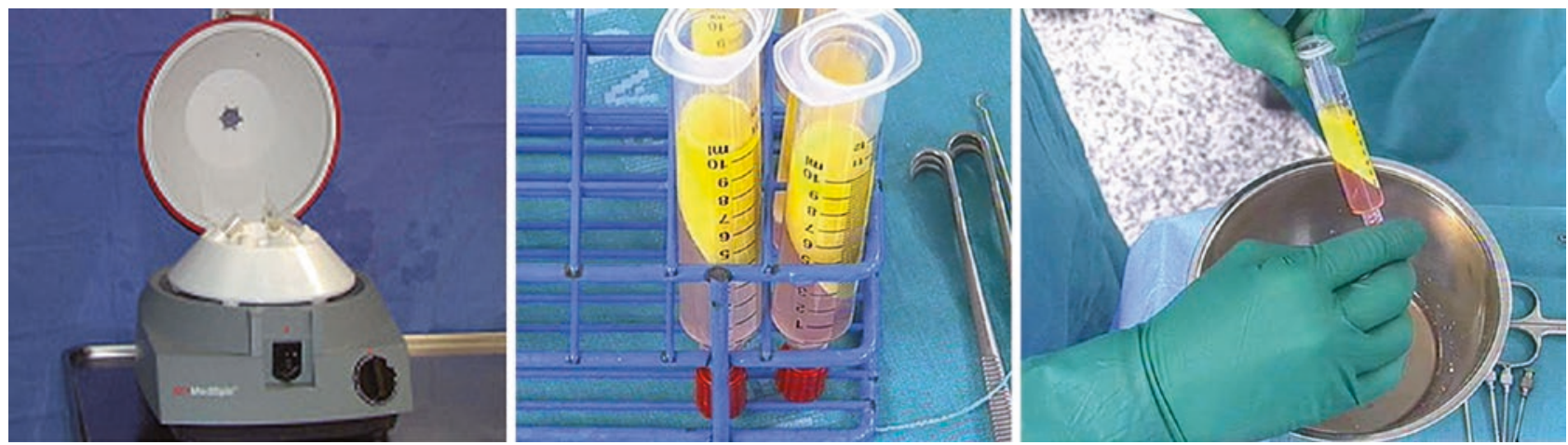

- Fig. 39.1 Centrifugation
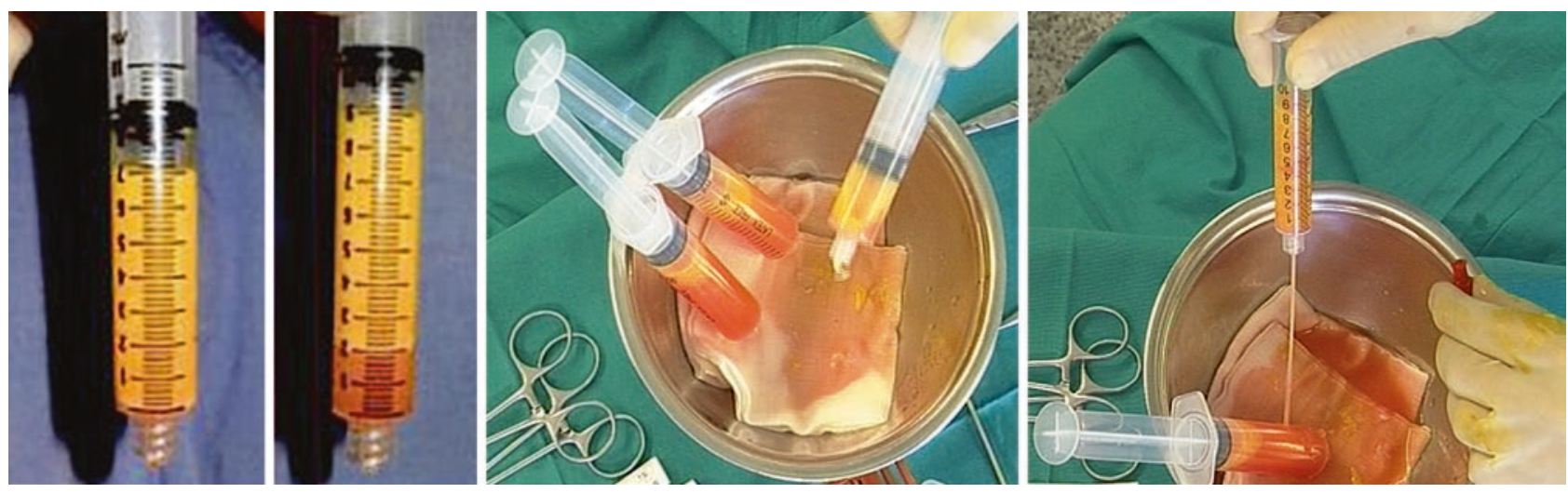

- Fig. 39.2 Decantation

ered a sizing procedure, nowadays the immediate centrifugation at low speed (3000 rpm for 3-5 minutes) is allowed, but decantation and mechanical purification are preferable, because they don't alter any features of the tissue (- Figs. 39.1 and 39.2).

3. The manufacturing of the graft may not involve combination with another article, except for water, crystalloids, or a sterilizing, preserving, or storage agent. Enzymatic process is not allowed. Separation and reinjection of the stromal vascular fraction are not allowed. Till some years ago, enzymes were permitted to be used as collagenase in order to "upgrade" an adipose stem cell (ASC)-poor fat. In fact, the collagenase could disrupt the link between the ASC permitting to add some freshly isolated stromal vascular fraction in order to obtain a so-called ASC-enriched fat. Nowadays, this enzymatic process is considered manipulation, so it cannot be allowed. Also, today it is possible to prepare the adipose tissue with a tumescent solution, and the lipoaspirate obtained can be added with sterile saline without altering the tissue. This process guarantees the keeping of the original features of the tissue, but it doesn't help us in increasing the engraftment of the free fat graft; so, as we will see later, we can use some ancillary procedures to increase the survival of the fat grafting, as plasma-rich platelet.
4. The graft has to be harvested with sterile technique; the contact with air has to be minimal. In order to perform this process, a so-called closed system has to be used that is featured by a sterile disposable canister for collecting, separating, concentrating, and transferring the fat graft (• Fig. 39.3).

5. The graft has to be taken with small cannulas (3-4 mm) and injected with 2-2.5 mm cannulas in a retrograde way (retrograde fat distribution). Actually this is not a new guideline; indeed it was there already in 1893 when Neuber A. treated scars noticing that small-dimension fat graft could be more effective than bigger one (• Fig. 39.4).

\subsection{The Procedure}

Set up by Coleman in 1925, in order to improve overall adipose cell survival, the procedure is featured by a tumescent technique lipo-aspiration with cannulas or micro cannulas from regions as, for example, the abdomen, medial thighs, hips, and trochanteric region. The fat taken can be centrifuged for $3000 \mathrm{rpm}$ for 3-5 minutes or decanted in order to separate the oily part, which can be calcified if injected, from the seric part and the "real" fat. After being separated, the adipose tissues are injected, with small cannulas, in other regions as, for example, the face. The lipofilling 

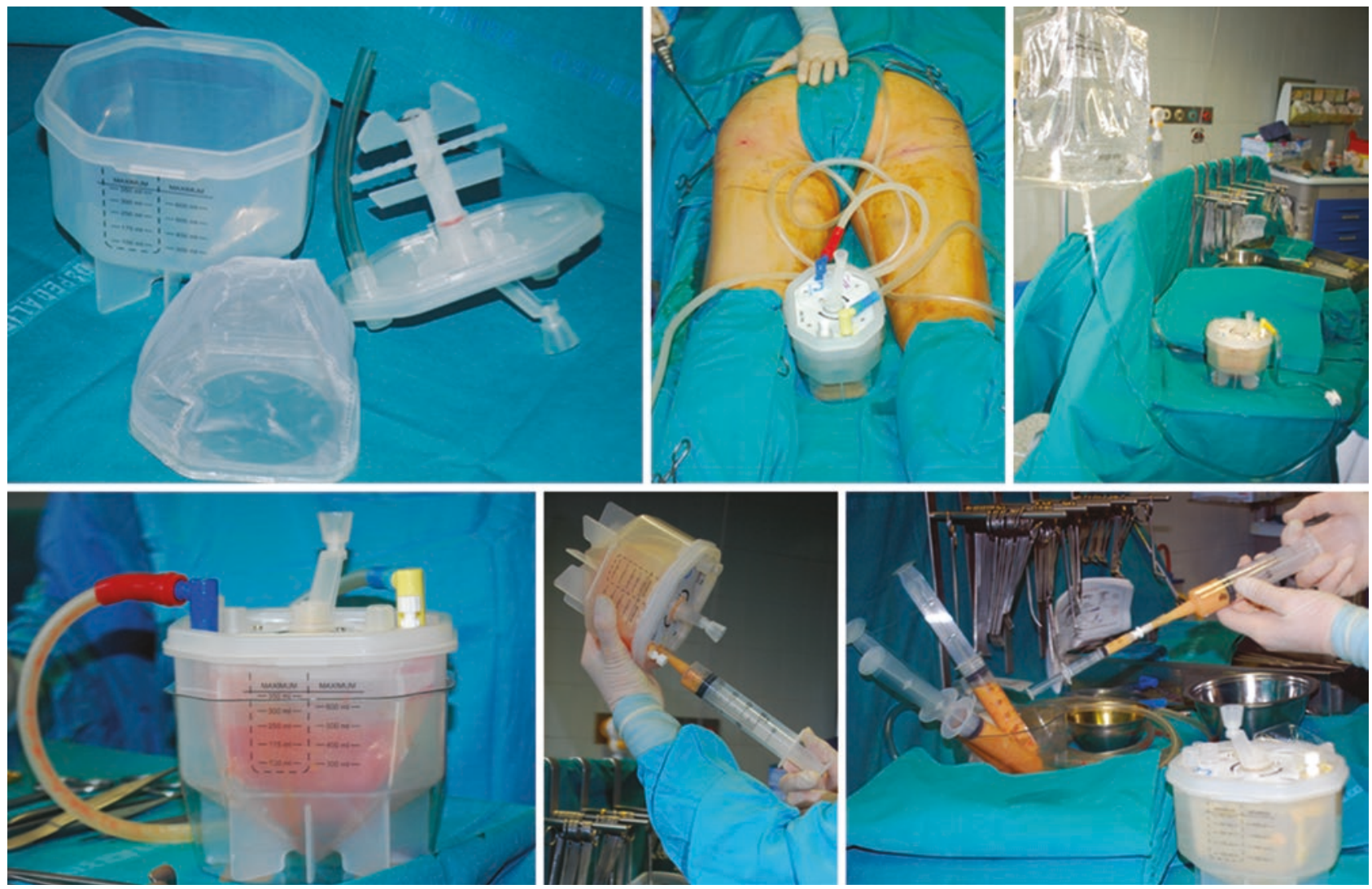

- Fig. 39.3 Closed system

- Fig. 39.4 The cannulas

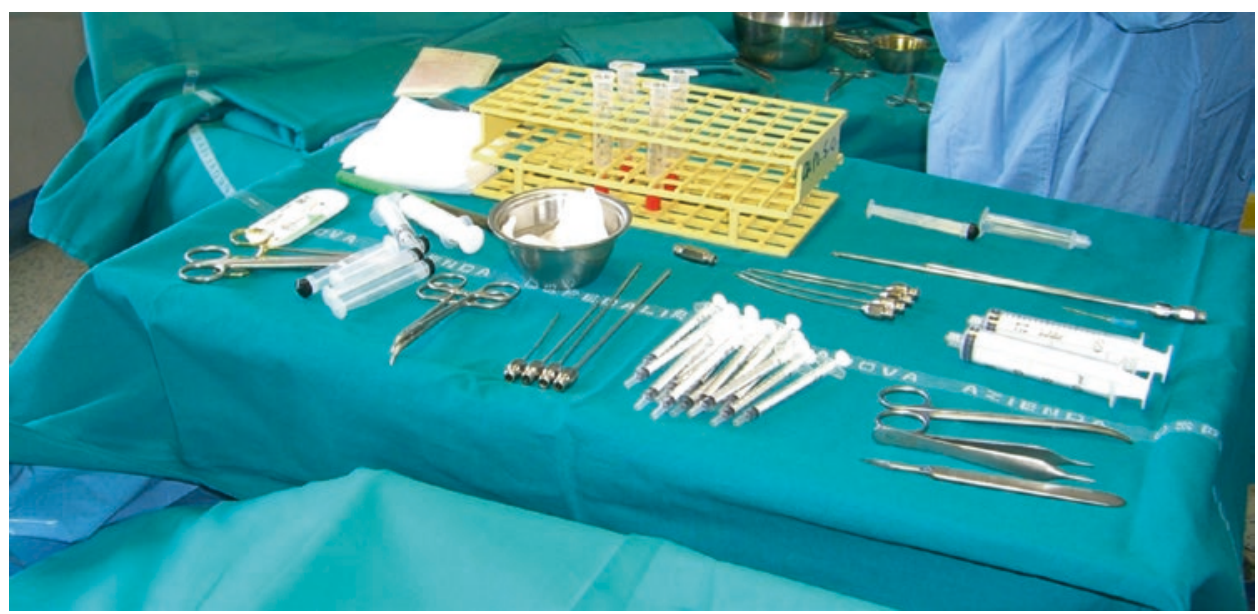

procedure can be executed under general or locoregional anesthesia; it depends on the volume of adipose tissue that we have to take and on the area that has to be injected. The procedure is not free of peri- and postoperative complications such as infection, hematoma, or calcifications.

It also has to be also considered that the free fat grafting can be physiologically reabsorbed. Unfortunately, the engraftment percentage is a subjective parameter, and it varies from $10 \%$ to $70 \%$ and in some comes till a total reabsorption. This feature can be brought to multiple sessions in order to obtain the correct volume.

\subsection{The Free Fat Grafting and Scars [3-9] (• Figs. 39.5, 39.6, 39.7, and 39.8)}

Considering its biological properties, we can propose the free fat grafting both to stimulate wound healing and to treat or prevent scarring, because it can act and improve more than one feature of a scar:

1. The skin texture, thickness, and pliability

2. The volume and contour

3. The fibrosis

4. The pain 


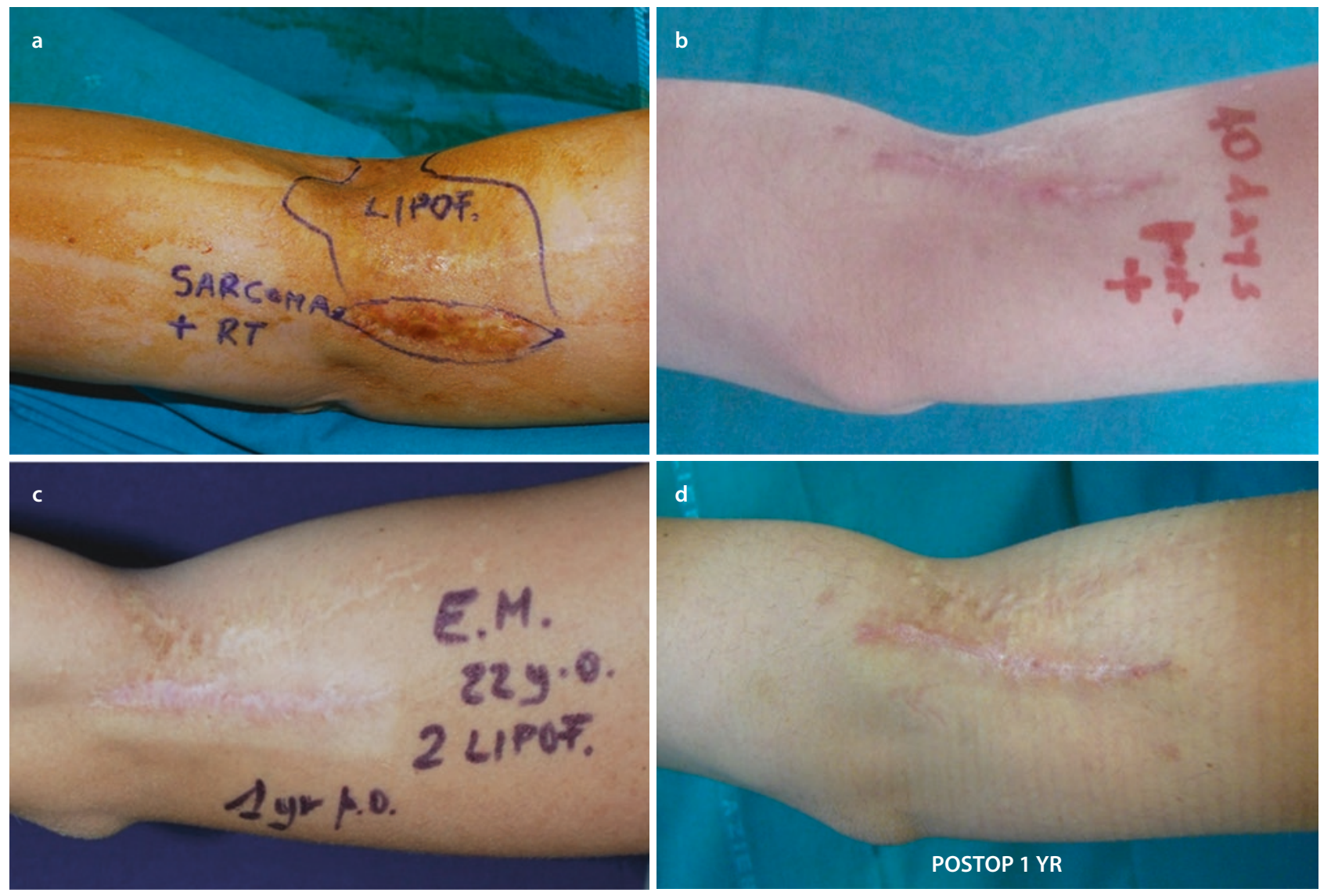

- Fig. 39.5 Skin texture and color: a Pre-lipofilling after radiotherapy for sarcoma; b 40 days postop; c preop second lipofilling; d postsecond lipofilling at 1 year
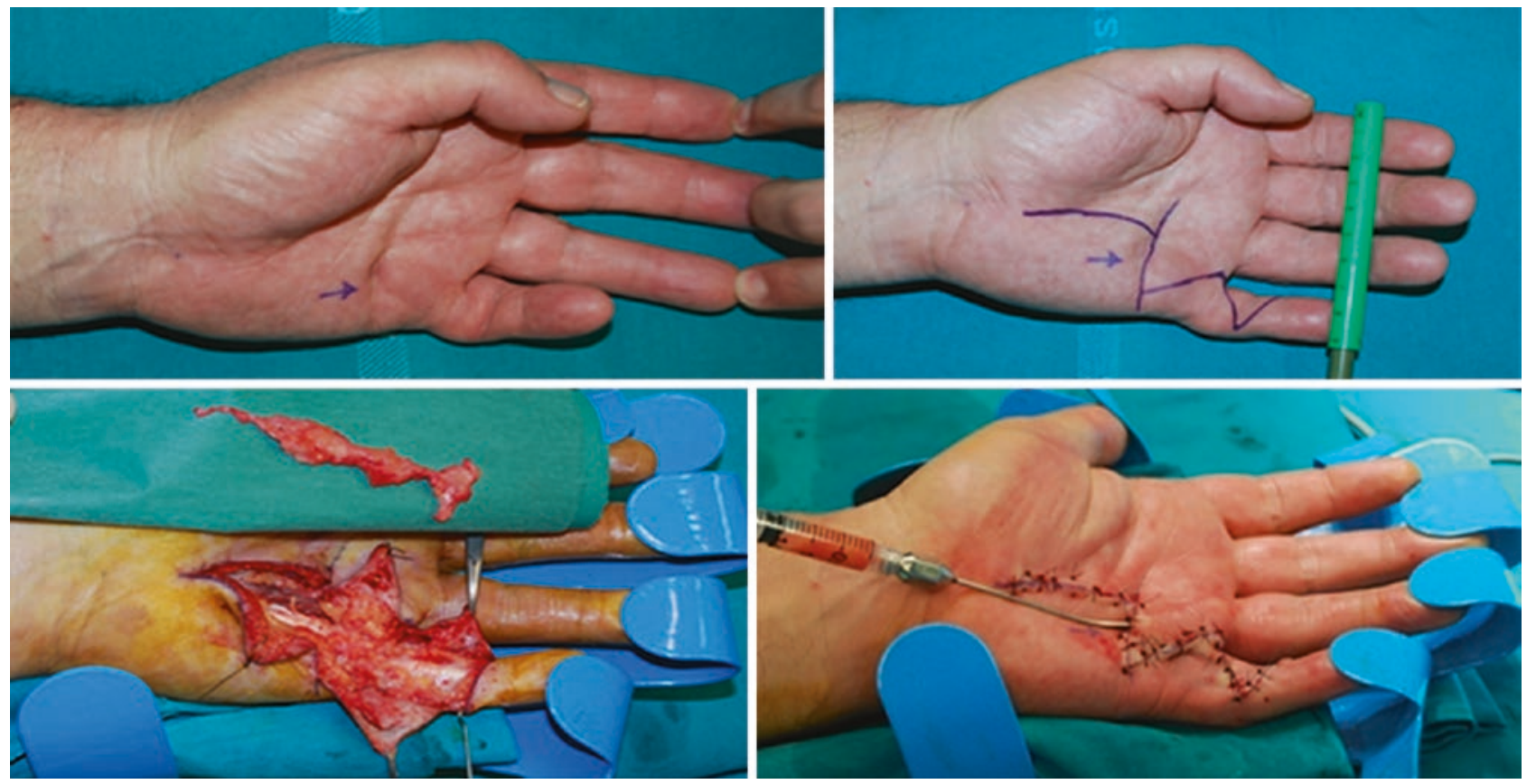

- Fig. 39.6 Fibrosis control in Dupuytren's disease 

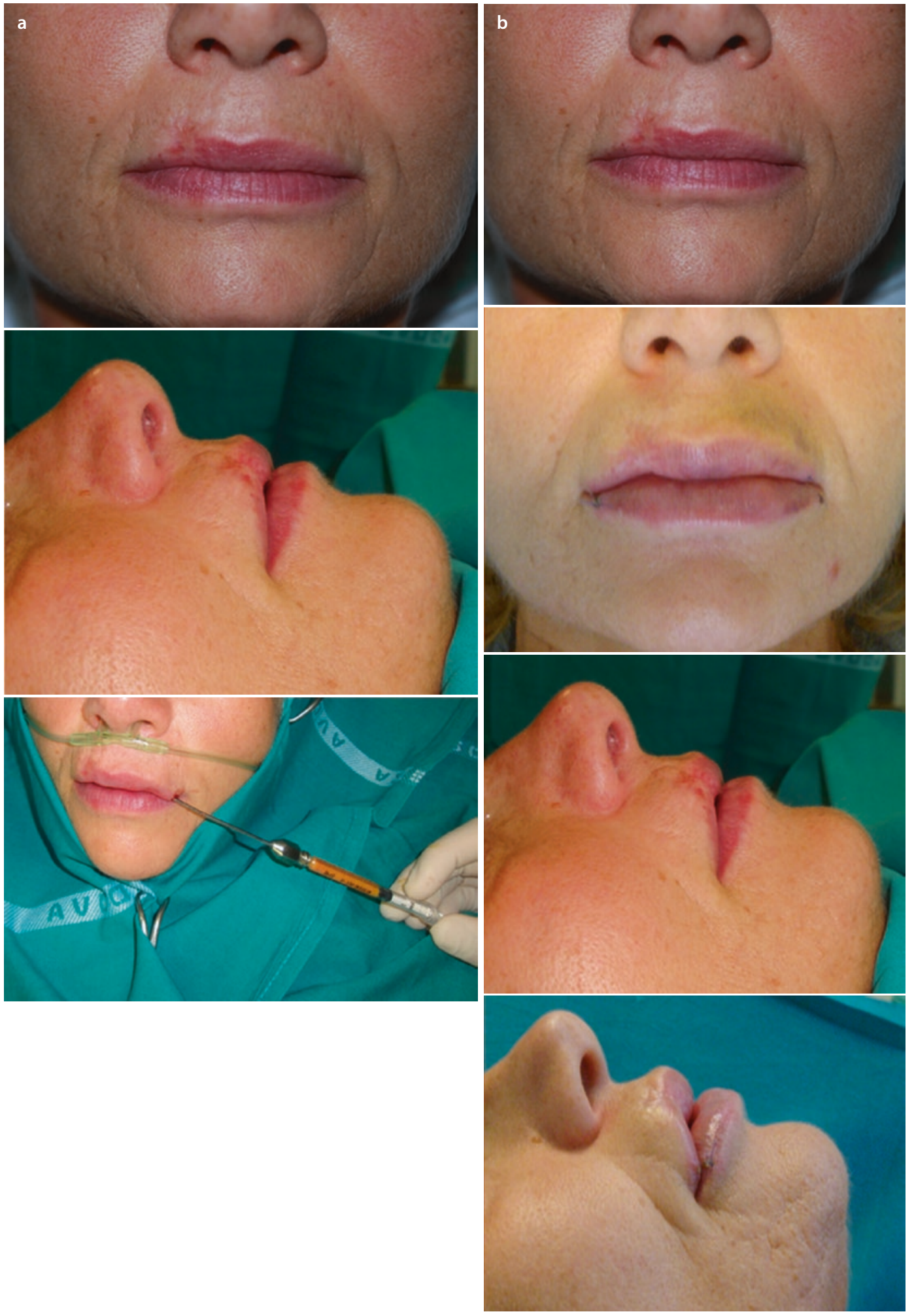

- Fig. 39.7 a Volume and contour: dog bite scar; pre-lipofilling; interoperative. b Volume and contour: postoperative results 

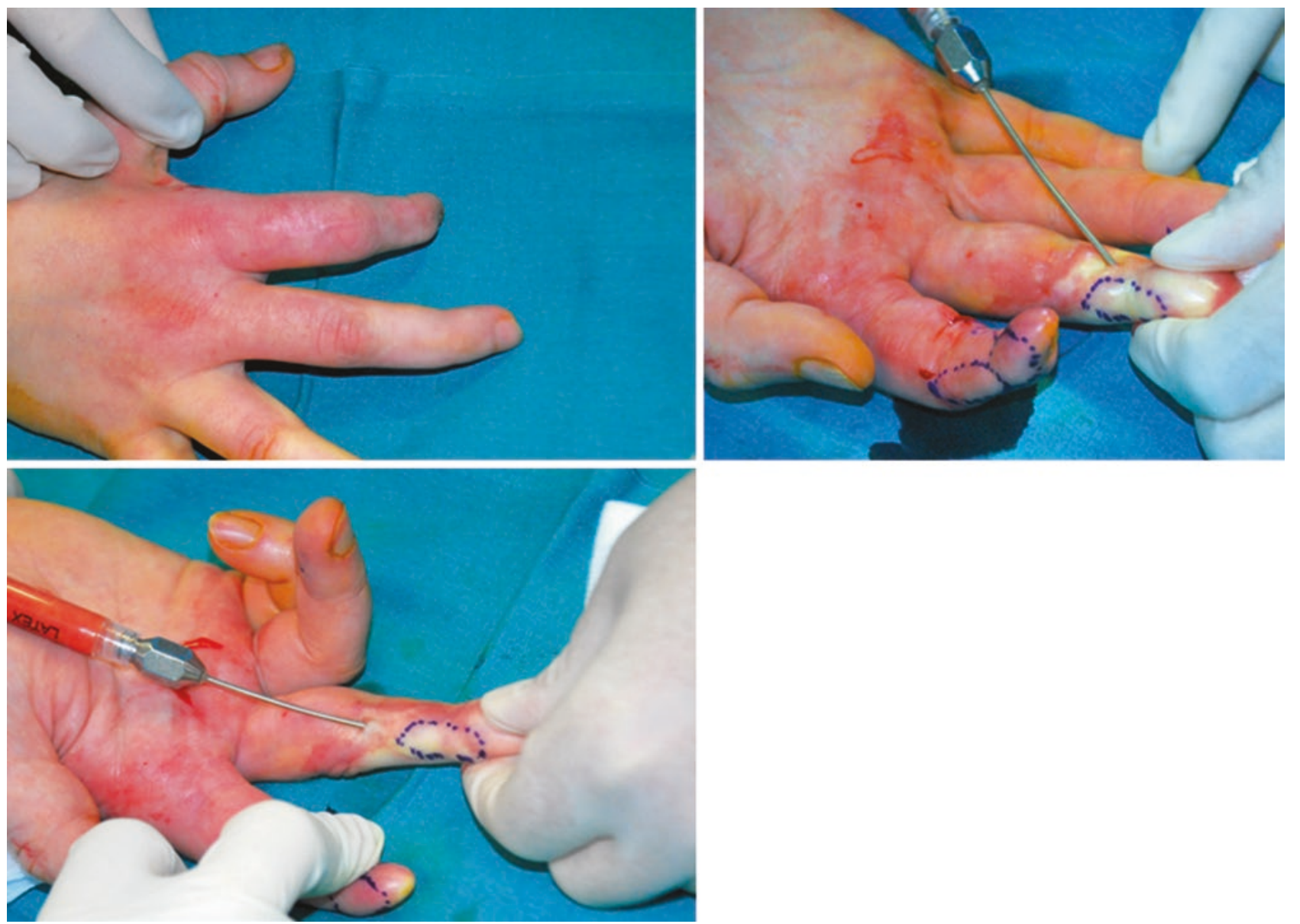

- Fig. 39.8 Pain and nerve release: posttraumatic nerve entrapment

\subsubsection{The Skin Texture, Thickness, and Pliability}

As demonstrated in 2009 by Mojallal et al. in an experimental mouse model, the transplantation of human adipose tissue to nude mice brought to an improving in the thickness of dermis. These results were obtained, thanks to a stimulated neosynthesis of type I collagen fibers, with a consequent composition of a denser extracellular dermal matrix. This skin feature improved its texture, thickness, and pliability.

Some years later, in 2013, Klinger M. et al. performed a study on humans, and they noted that the areas treated with free fat grafting assumed features that were similar to normal skin in terms of elasticity and softness. In order to demonstrate these features, they also used a durometer evaluation that demonstrated a significant reduction of skin hardness.

But why do ADSCs behave this way? As we said earlier, ADSCs can modulate and reduce inflammation; studies demonstrated that there is also an improvement in melanin secretion. These last utilities can also modify the skin color, thereby reducing the erythema and improving normal color of the skin.

\subsubsection{The Fibrosis}

Related to skin thickness, fibrosis can be due to diseases as, for example, connective tissue diseases or Dupuytren's disease or treatment such as radiotherapy. We used lipofilling in the treatment of a specific connective tissue disease called scleroderma or systemic sclerosis. This systemic connective tissue disease is featured by a progressive skin fibrosis especially on the face and in particular in the perioral region which brings to the so-called microstomia. The patients affected (usually female) report a bad lips sensation and progressive mouth dryness, difficulty in speaking, oral hygiene, and feeding. In our experience, the application of fat grafting 
in perioral region has improved the symptoms reducing skin stiffness and improving opening the mouth.

Regarding the use of lipofilling in Dupuytren's disease, this pathology is due to a fibrotic degeneration of the longitudinal fibers of palmar aponeurosis, with the consequent involvement of subcutaneous septa, the loss of subcutaneous fat, and the adherence of dermis to palmar aponeurosis. In this case the autologous adipose tissue is used as adjuvant therapy to prevent the formation of new fibrotic tissue (• Fig. 39.7).

Finally, regarding radiotherapy, the fibrosis is a sort of adverse event that rises years after the end of radiotherapy treatment and that it is due to the presence of an important post irradiated fibro-necrotic tissue featured by local dystrophic fat lobules, smaller but thicker vessels, and a perivascular fibrosis. In this case the fat grafting reduces the necrotic areas and improves neoangiogenesis with a consequent increasing in skin quality. It is supposed that these results are due to the intrinsic ability of the ADSCs to secrete growth factors, as angiogenic ones, as we said earlier.

\subsubsection{The Volume and Contour}

As we said earlier, the free fat grafting has a trophic/volumetric effect. As Mojallal noticed the autologous free fat grafting has to be considered as a dynamic filler. In fact, the "fat filler" doesn't have only an immediate effect of restoring volume, but it has the ability to produce adipogenesis with the consequent transformation of the preadipocytes in mature adipocytes. This can bring to an increased volume of the soft adipose tissue. The plasticity that features the adipose tissue makes it a good filler to restore the contour too, not only in posttraumatic scars but also in postoperative deformities and/or congenital malformation or adult progressive and selflimited deformation as, for example, in Parry-Romberg syndrome (the adult hemifacial atrophy).

\subsubsection{Pain: The Analgesic Effect}

According to Coleman who demonstrated the positive effect of free tissue transfer on neuralgias, Riyat et al. published in 2017 a review in which they noticed as the lipofilling had an analgesic effect, demonstrated with tests as, for example, POSAS or McGill Pain Questionnaire, on 966 patients. This effect seems to be due to multiple aspects: first of all, there is a mechanical release. To perform the injection, we have to insert the cannula under the scar in the space of the dermo-hypodermal junction, and then we proceed to distribute the fat in a retrograde way to reproduce a sort of a weblike matrix. This procedure can mechanically release fibrotic adherence and nerve entrapment, but most of all, the presence of the adipose stem cells can stimulate the nerve repair mediated by the brain-derived neurotrophic factor (BDNF), an adipose-derived neurotrophin which is fundamental in nerve growth and repair. But this is not the only mechanism to improve analgesic effect: as we said earlier, fat tissues are featured by an anti-inflammatory effect which is mediated both by the TGF $\beta$ production that can modulate and suppress T-cell function and by the production of IL 10 a cytokine which is abundantly produced by ADSCs and is known to inhibit $\mathrm{Cd} 4$ and CD8 lymphocytes reducing the inflammation response.

\subsubsection{The Possible Applications}

Based on the aforementioned effects, we can propose the free fat grafting for the treatment and prevention of multiple kinds of scars such as the following:

1. The treatment of atrophic and/or depressed scar, for example, in case of acne or previous removal of bulky neoformations. In case of atrophic and/or depressed scar, the free fat grating can be used both for a volumetric effect, indeed it fills the empty spaces, and for regenerative effect, indeed it can improve the skin texture.

2. The treatment of retracting scars which can limit not only the aesthetic point of view but also many functions as the flexor extension of the neck or of the elbow. In this case we can associate fat grafting with surgery as z-/w-plasty or flaps.

3. Unstable posttraumatic scars in order to obtain both scar stabilization and to improve wound healing.

4. Fibrotic diseases such as Dupuytren's disease and connective tissue diseases such as scleroderma, as we said previously.

5. Fibrotic irradiated tissue.

6. Fibrotic response to foreign bodies named periprosthetic fibrotic and, often, contracted capsule: this response can be described as a pathological and excessive deposition of collagen fibers around a mammary implant, in this case, the lipofilling.

7. Postsurgical deformities.

8. Nerve release in case of neuromas or posttraumatic pain.

\subsection{The Ancillary Procedures to Increase the Overall Survival of Adipose Cells}

\subsubsection{The Plasma-Rich Platelet [10]}

Known as PRP, it is a product obtainable by a blood draw of at least $20 \mathrm{cc}$. It is featured by a high concentration of platelet $>300-350 \times 10^{3}$ platelets $\mu \mathrm{L}$, (three to five times than normal) and the production of growth factors, including $P D G F, T G F \beta, V E G F, I G F-1, F G F$, and $E G F$, released by the $\alpha$ granules of the activated 
platelets. These factors promote tissue repair, modulate inflammatory processes and neoangiogenesis, and ultimately regulate homeostasis of tissue and regenerative process. The PRP is easily obtainable without morbidity for the patient, and it can be associated to free fat grafting during the same session, in order to increase the percentage of engraftment and to improve the overall adipose cell survival.

\subsubsection{The External Volume Expansion [11]}

In order to improve on-site adipogenesis and overall adipose cell survival, a new technique called external volume expansion (EVE) is being used in these years. This procedure is based on tissue expansion combined with the mechanobiology principles which explain how mechanical forces, such as the ones produced by the application of the negative pressure therapy, can be transduced in the cells in order to stimulate processes as cellular shaping, proliferation, and differentiation. In fact, studies demonstrated that the EVE application can increase cell proliferation, deep dermis capillary density, and adipogenesis due to the edema and inflammation response which are proadipogenic factors.

\subsubsection{The Future}

Recently the fat graft has been proposed for the treatment of keloids or hypertrophic scars. Keloid is a fibroproliferative disorder which is known as pathological scar, and it is featured by an excessive production and deposition of extracellular dermal matrix due to prolonged inflammatory and proliferative phases of wound healing. Nowadays, there is not a gold standard treatment for pathological scars; it is actually based both on prevention with compressive garments or silicone gel or sheets both on topical injection of keloid with anti-inflammatory drugs as corticosteroids. Based on the anti-inflammatory properties of the free fat grafting and on its ability to stimulate neoangiogenesis and a better skin quality, it has been proposed to use it in the treatment of hypertrophic/keloid scars, for example, in postburn patient. Even if the first impressions are absolutely positive, unfortunately, as Lee $\mathrm{G}$ et al. noted in 2017, there is a lack of high-level literature about this field, so further studies are required.

\subsection{Something to Discuss: The Oncological Point of View}

Even if the adipose tissue transfer has multiple benefit effects, recently it has been supposed that the pluripotent adipose-derived stem cells and the neoangiogen- esis stimulated by growth factors can bring to the onset of a new neoplasia or a recurrence if the free fat graft is injected in a recipient site which is recently affected by a cancer, especially in a breast that has undergone a nipple-sparing mastectomy or a partial mastectomy (quadrantectomy). Many studies have been performed in order to demonstrate the safety of the fat grafting in such patients, but nowadays, even if a correlation between lipofilling and neoplasm or its recurrence has not been demonstrated, it is preferable to propose the free fat transfer after 2 years free of disease.

\subsection{Conclusion}

In light of our experience, the free fat grafting is considered an effective and promising treatment for scars. The biological properties of ADSCs combined with an easy and quick procedure, a low morbidity for the patients, and a high compliance make lipofilling an ideal treatment to propose. Unfortunately, the treatment has some limitations. First of all it cannot be proposed to a very slim patients; in fact we need fatty areas to obtain ADSCs; secondly, as we said earlier, the percentage of reabsorption of fat grafting subjectively varies from $10 \%$ to $70 \%$ till the complete reabsorption in extreme cases. This negative feature can result in multiple treatment sessions in order to obtain the right volume, but unfortunately it is not possible to set the number of sessions required since the beginning.

Fortunately, new studies and new research on cell therapy help us to avoid or reduce this problem, as we saw that the use of the external negative pressure therapy and/or the combination of free fat grafting with the plasma-rich platelet can improve the fat engraftment and the overall adipose cell survival with consequent better morpho-functional results.

\footnotetext{
- Take Home Messages

- The fat grafting contains stem cells called adiposederived stem cells.

- The adipose-derived stem cells are pluripotent cells.

- The fat grafting is an easy procedure with low morbidity rate.

- The fat injection has both a volumetric and a regenerative effect.

- The fat injection stimulates neo-adipogenesis and neoangiogenesis, and it modulates inflammation and fibrosis.

- The fat grafting can be reabsorbed.

- We can use external volume expansion and/or the combination with plasma-rich platelet to improve engraftment and overall adipose cell survival.
} 


\section{References}

1. US Department of Health and Human Services. Tissue guidances: human cells, tissues, and cellular and tissue-based products (hct/ps) from adipose tissue: regulatory considerations; draft guidance. www.fda.gov/BiologicsBloodVaccines/GuidanceComplianceRegulatoryInformation/Guidances/Tissue/ ucm427795.htm. Accessed 8 June 2015.

2. American Society of Plastic Surgeons and the Plastic Surgery Foundation. Docket \#FDA-2014-D-1856 (HCT/Ps). www. plasticsurgery.org/Documents/Legislation-Advocacy/Regulatory/ asps-response-hctp-draft-guidance.pdf. Accessed 7 July 2015.

3. Bassetto F, et al. Adipose-derived stem cells to modulate scar tissue: from biological basis to clinical application. In: Shiffman MA, Di Giuseppe A, editors. Stem cells in aesthetic procedures. art, science and clinical techniques. Berlin: Springer; 2014.
4. Bassetto F, et al. Fat grafting in the treatment of skin fibrosis and scars. J Wound Technol. 2014;24:17-19.

5. Mojallal A, et al. Improvement of skin quality after fat grafting: clinical and observation and an animal study. Plast Reconstr Surg. 2009;124:765.

6. Lee $\mathrm{G}$, et al. Autologous fat grafting in keloids and hypertrophic scars: a review. Scars Burn Heal. 2017;3:2059513117700157.

7. Riyat $\mathrm{H}$, et al. Autologous fat grafting for scars, healing and pain: a review. Scars Burn Heal. 2017;3:2059513117728200.

8. Bassetto F, et al. Fat grafting in wound healing and scar control. J Wound Technol. 2016;31:54-55.

9. Klinger M, et al. Autologous fat graft in scar treatment. J Craniofac Surg. 2013;24:1610Y1615.

10. Osaid H Alser, Ioannis Goutos (2018). The evidence behind the use of platelet-rich plasma (PRP) in scar management: a literature review. Scars, Burns \& Healing 4:205951311880877.

11. Lujan Hernandez J, et al. Induction of adipogenesis by external volume expansion. Plast Reconstr Surg. 2016;137(1):122-31.

Open Access This chapter is licensed under the terms of the Creative Commons Attribution 4.0 International License (http://creativecommons. org/licenses/by/4.0/), which permits use, sharing, adaptation, distribution and reproduction in any medium or format, as long as you give appropriate credit to the original author(s) and the source, provide a link to the Creative Commons license and indicate if changes were made.

The images or other third party material in this chapter are included in the chapter's Creative Commons license, unless indicated otherwise in a credit line to the material. If material is not included in the chapter's Creative Commons license and your intended use is not permitted by statutory regulation or exceeds the permitted use, you will need to obtain permission directly from the copyright holder. 